Pacific Journal of Mathematics

SETS OF CONSTANT WIDTH

Vol. 19, No. 1

May 1966 


\title{
SETS OF CONSTANT WIDTH
}

\author{
G. D. ChAKERIAN
}

A lower bound, better than those previously known, is given for the volume of a 3-dimensional body of constant width 1. Bounds are also given in the case of $n$-dimensional bodies of constant width $1, n \geqq 4$. Short proofs of the known sharp bounds for such bodies in the Euclidean and Minkowskian planes are given using properties of mixed areas. An application is made to a measure of outer symmetry of sets of constant width in 2 and 3 dimensions.

Let $K$ be a convex body in $n$-dimensional Euclidean space $E_{n}$. For each point $u$ on the unit sphere $S$ centered at the origin, let $b(u)$ be the distance between the two parallel supporting hyperplanes of $K$ orthogonal to the direction. The function $b(u)$ is the "width function" of $K$. If $b(u)$ is constant on $S$, then we say $K$ is a body of constant width.

If $K_{1}$ and $K_{2}$ are convex bodies, then $K_{1}+K_{2}$ is the "Minkowski sum" or "vector sum" of $K_{1}$ and $K_{2}$ [5, p. 79]. The following useful theorem is well-known.

THEOREM 1. A convex body $K$ has constant width $b$ if and only if $K+(-K)$ is a spherical ball of radius $b$.

In the case of $E_{2}$, a number of special properties of sets of constant width are known-for example, the following theorem of Pàl (see [5, p. 127]).

THEOREM 2. Any plane convex body $B$ of constant width admits a circumscribed regular hexagon $H$.

We shall be concerned with the following type of result, due to Blaschke and Lebesgue (see [1], [3], [4], [5, p. 128], [9]).

THEOREM 3. Any plane convex body $B$ of constant width 1 has area not less than $(\pi-\sqrt{3}) / 2$, the area of a Reuleaux triangle of width 1.

The following short proof of Theorem 3 will set the stage for some later arguments.

Proof of Theorem 3. Let $A(K)$ denote the area of $K$. The "mixed area" of the plane convex bodies $K_{1}$ and $K_{2}, A\left(K_{1}, K_{2}\right)$, can be 
defined by the fundamental relation [5, p. 48],

$$
A\left(K_{1}+K_{2}\right)=A\left(K_{1}\right)+2 A\left(K_{1}, K_{2}\right)+A\left(K_{2}\right) .
$$

The mixed area is monotonic in each argument $[5, \mathrm{p} .86]$. That is, if $K_{1} \subset K_{2}$, then

$$
A\left(K_{1}, K\right) \leqq A\left(K_{2}, K\right) .
$$

It follows from (1), setting $K_{1}=K_{2}=K$, that

$$
A(K, K)=A(K) \text {. }
$$

Now let $H$ be a regular hexagon circumscribed about $B$ (Theorem 2). Assume the center of $H$ is the origin, so $H=-H$. Then, using (2) and (3), we obtain

$$
A(B,-B) \leqq A(H,-H)=A(H, H)=A(H) .
$$

Thus, by (4), (1), and Theorem 1, we have

$$
\begin{aligned}
\pi & =A(B+(-B))=2 A(B)+2 A(B,-B) \\
& \leqq 2 A(B)+2 A(H)=2 A(B)+\sqrt{3},
\end{aligned}
$$

from which the theorem follows.

It has long been conjectured that in $E_{3}$ any convex body of constant width 1 has volume at least that of a certain "tetrahedron of constant width" $T$ (see [12, p. 81] for the construction of $T$ ). A computation of the volume of $T$ leads to the conjecture,

Conjecture 1. Any 3-dimensional convex body of constant width 1 has volume not less than

$$
\frac{2 \pi}{3}-\frac{\pi \sqrt{3}}{4} \cos ^{-1}(1 / 3) \approx .42 .
$$

In $\S 2$ we shall prove that if $B_{3}$ is a 3 -dimensional body of constant width 1 , with volume $V\left(B_{3}\right)$, then

$$
V\left(B_{3}\right) \geqq \beta=\frac{\pi}{3}(3 \sqrt{6}-7) \approx .365 .
$$

Our proof of (6) will depend upon the following theorem of Blaschke [2].

THEOREM 4. If a 3-dimensional convex body of constant width $b$ has volume $V$ and surface area $S$, then

$$
2 V=b S-\frac{2 \pi}{3} b^{3} .
$$


It follows from (7) that Conjecture 1 is equivalent to:

Conjecture 1'. Any 3-dimensional convex body of constant width 1 has surface area not less than

$$
2 \pi-\frac{\pi \sqrt{3}}{2} \cos ^{-1}(1 / 3) \text {. }
$$

Conjecture 1 can be transformed into still another form using the concept of "mixed surface area." Let $S(K)$ denote the surface area of $K$. If $K_{1}$ and $K_{2}$ are 3-dimensional convex bodies, then the surface area of $K_{1}+K_{2}$ can be written in the form

$$
S\left(K_{1}+K_{2}\right)=S\left(K_{1}\right)+2 S\left(K_{1}, K_{2}\right)+S\left(K_{2}\right),
$$

where $S\left(K_{1}, K_{2}\right)$ is the mixed surface area. Thus, if $K$ has constant width $1,4 \pi=S(K+(-K))=2 S(K)+2 S(K,-K)$. Hence Conjectures 1 and $1^{\prime}$ are equivalent to:

Conjecture 1". Any 3-dimensional convex body of constant width 1 has mixed surface area not greater than

$$
\frac{\pi \sqrt{3}}{2} \cos ^{-1}(1 / 3) \text {. }
$$

Firey [6] has proved that the volume $V$ of an $n$-dimensional convex body of constant width 1 satisfies

$$
V \geqq \frac{\pi-\sqrt{3}}{n !}, n \geqq 2 \text {. }
$$

In $\S 2$ we give the generally better lower bound,

$$
V \geqq \lambda \omega_{n} \prod_{k=3}^{n}\left(1-\sqrt{\frac{k}{2 k+2}}\right), n \geqq 3,
$$

where $\omega_{n}$ is the volume of the unit ball in $E_{n}$, and

$$
\lambda=\frac{\pi-\sqrt{3}}{2 \pi} \text {. }
$$

Let $C$ be a centrally symmetric convex body centered at the origin in $E_{n}$. Then $C$ is the unit sphere for a Minkowskian geometry. We say that a body $K$ has "constant width relative to $C$ " if $K+(-K)$ is homothetic to $C$. In particular, one says that $K$ and $C$ are "equivalent in width" in case $K+(-K)=2 C$, since the condition implies that $K$ and $C$ have the same width function. When $C$ is the ordinary unit sphere we obtain the ordinary sets of constant width. Results about 
plane sets of relative constant width analogous to Theorem 2 and 3 are known (see [8], [10], and [11]). In $\S 3$ we give a proof of the analogue of Theorem 3 in the Minkowski plane, using the same method as in our proof of Theorem 3 .

Section 4 is devoted to some results on measures of outer symmetry for sets of constant width.

2. Proof of (6). Let $B_{3}$ be a 3-dimensional convex body of constant width 1 . Then the inscribed sphere of $B_{3}$ has radius $\geqq$ $1-\sqrt{3 / 8}$ (see [5, p. 125]). Assume that the center of the inscribed sphere is the origin. If $p(u)$ is the supporting function of $B_{3}$, then we have $p(u) \geqq 1-\sqrt{3 / 8}$. Hence,

$$
3 V\left(B_{3}\right)=\int_{B_{3}} p(u) d S(u) \geqq(1-\sqrt{3 / 8}) S\left(B_{3}\right),
$$

where $S\left(B_{3}\right)$ is the surface area of $B_{3}$. Using Theorem 4 in (10), we obtain

$$
3 V\left(B_{3}\right) \geqq(1-\sqrt{3 / 8})\left(2 V\left(B_{3}\right)+\frac{2 \pi}{3}\right),
$$

and (6) follows upon solving (11) for $V\left(B_{3}\right)$. This completes the proof.

Proof of (9). Define

$$
\lambda_{n}=\inf V(K),
$$

as $K$ ranges over all bodies of constant width 1 in $E_{n}$, and $V(K)$ is the volume of $K$. The Blaschke selection principle implies that there exist bodies of constant width 1 having volume $\lambda_{n}$. Let $B$ be such a body, and let $p(u)$ be the support function of $B$ with the center of its inscribed sphere as origin. Then, by [5, p. 125],

$$
p(u) \geqq 1-\sqrt{\frac{n}{2 n+2}} .
$$

Denoting the area element of $B$ by $d S(u)$, we have,

$$
n \lambda_{n}=n V(B)=\int_{B} p(u) d S(u) \geqq\left(1-\sqrt{\frac{n}{2 n+2}}\right) S(B),
$$

where $S(B)$ is the surface area of $B$. If we denote by $B_{u}$ the projection of $B$ onto a hyperplane orthogonal to $u$, then (see [5, p. 89])

$$
S(B)=\frac{1}{\omega_{n-1}} \int V\left(B_{u}\right) d u,
$$


where $V\left(B_{u}\right)$ is the $(n-1)$-dimensional volume of $B_{u}$ and the integration is over the surface of the unit sphere in $E_{n}$. Since $B_{u}$ is an $(n-1)$-dimensional body of constant width 1 , we have by (12) that $V\left(B_{u}\right) \geqq \lambda_{n-1}$. Hence

$$
S(B) \geqq \frac{n \omega_{n} \lambda_{n-1}}{\omega_{n-1}} .
$$

Combined with (13), this yields

$$
\lambda_{n} \geqq\left(1-\sqrt{\frac{n}{2 n+2}}\right) \frac{\omega_{n}}{\omega_{n-1}} \lambda_{n-1},
$$

from which (9) follows. This completes the proof.

3. In this section, $C$ is a centrally symmetric plane convex body centered at the origin $0 . C$ admits an inscribed affine regular hexagon $H$ (i.e., the affine image of a regular hexagon) having a side parallel to any specified direction [10]. Let the vertices of $H$ be labelled $P_{1}, P_{2}, \cdots, P_{6}$ on the boundary of $C$ traversed in the positive direction. A "relative Reuleaux triangle" is obtained by attaching $\operatorname{arcs} P_{1} P_{2}, P_{3} P_{4}$, and $P_{5} P_{6}$ of the boundary of $C$ to the respective sides $P_{1} P_{2}, P_{2} 0,0 P_{1}$ of the triangle $0 P_{1} P_{2}$. With $H$ as above, a centrally symmetric hexagon circumscribed about $C$ and touching $C$ at $P_{i}, 1 \leqq i \leqq 6$, is called a " $C$-hexagon." In fact, any hexagon homothetic to such a hexagon will be called a $C$-hexagon. Note that it case $C$ is a circle, any $C$ hexagon is just a regular hexagon. One then sees that the following theorem from [10] is a Minkowskian geometry analogue of Theorem 2.

Theorem 2'. Let $K$ be equivalent in width to $C$. Then $K$ admits a circumscribed C-hexagon.

Let $H$ be a $C$-hexagon circumscribed about $C$. Let $H^{\prime}$ be the corresponding affine regular hexagon inscribed in $C$ with its vertices on $H$. Then we shall show that

$$
A(H) \leqq 4 / 3 A\left(H^{\prime}\right) \text {. }
$$

This follows from the following general lemma.

LEMma 1. Let $H^{\prime}$ be an affine regular hexagon inscribed in a centrally symmetric plane convex body $K$. Then

$$
A(K) \leqq 4 / 3 A\left(H^{\prime}\right)
$$

Proof. By considering the support lines of $K$ through the vertices of $H^{\prime}$, one sees that it suffices to prove (18) for $K$ a centrally 


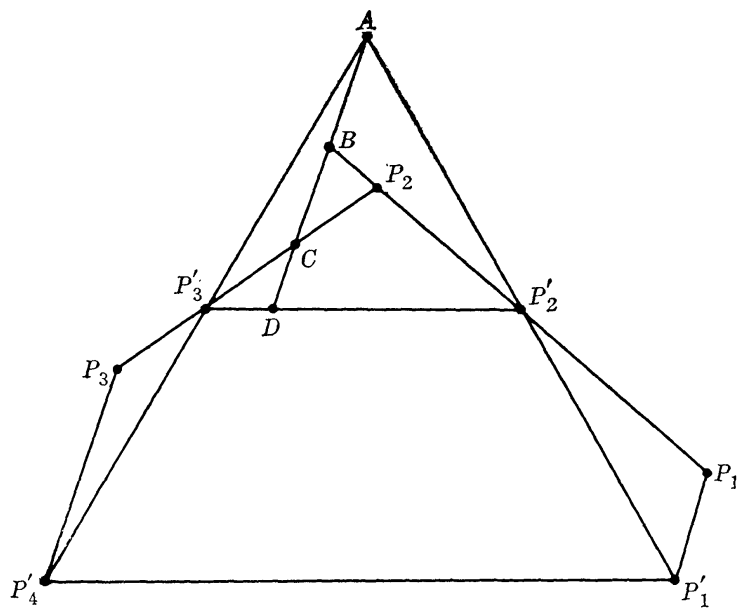

Figure 1.

symmetric hexagon $H$. Since the problem is affine invariant, one may even assume $H^{\prime}$ is a regular hexagon, although this does not really simplify matters. In Figure $1, P_{1}^{\prime}, P_{2}^{\prime}, P_{3}^{\prime}, P_{4}^{\prime}$ are consecutive vertices of $H^{\prime}$, and $P_{1}, P_{2}, P_{3}$ are vertices of $H . A D$ is drawn parallel to $P_{4}^{\prime} P_{3}$, which is parallel to $P_{1}^{\prime} P_{1}$ (the degenerate cases, where $P_{3}=P_{4}^{\prime}$ or $P_{1}=P_{1}^{\prime}$ are easily disposed of and will not be dwelt upon here). $B$ is the intersection of $P_{1} P_{2}$ with $A D$, and $C$ is the intersection of $P_{3} P_{2}$ with $A D$. Triangle $P_{4}^{\prime} P_{3} P_{3}^{\prime}$ is congruent to $P_{3}^{\prime} C A$, and $P_{1}^{\prime} P_{1} P_{2}^{\prime}$ is congruent to $P_{2}^{\prime} B A$. Hence the area of the pentagon $P_{1}^{\prime} P_{1} P_{2} P_{3} P_{4}^{\prime}$ is not greater than the area of triangle $P_{1}^{\prime} A P_{4}^{\prime}$, so the area of $H$ is not greater than twice the area of $P_{1}^{\prime} A P_{4}^{\prime}$, which is precisely $4 / 3 A\left(H^{\prime}\right)$. This completes the proof.

TheOREM 3'. Any plane convex body $K$ which is equivalent in width to $C$ has area not less than that of some relative Reuleaux triangle equivalent in width to $C$.

Proof. It is easy to check that the area of any relative Reuleaux triangle $T$ equivalent in width to $C$ is given by

$$
A(T)=2 A(C)-4 / 3 A(H),
$$

where $H$ is the affine regular hexagon inscribed in $C$ on which the construction of $T$ is based. Let $H^{\prime}$ be a $C$-hexagon circumscribed about $K$ (Theorem $2^{\prime}$ ), let $H^{\prime \prime}$ be the translate of $H^{\prime}$ circumscribed about $C$, and let $H$ be the corresponding affine regular hexagon inscribed in $C$ with its vertices on $H^{\prime \prime}$. Let the center of $H^{\prime}$ be at the origin (which can be assumed by translating $K$ ) so $H^{\prime}=-H^{\prime}$. Then, 
proceeding as in the proof of Theorem 3, and using (17), we have

$$
\begin{aligned}
4 A(C) & =A(K+(-K))=2 A(K)+2 A(K,-K) \\
& \leqq 2 A(K)+2 A\left(H^{\prime},-H^{\prime}\right)=2 A(K)+2 A\left(H^{\prime}\right) \\
& =2 A(K)+2 A\left(H^{\prime \prime}\right) \leqq 2 A(K)+8 / 3 A(H) .
\end{aligned}
$$

Hence,

$$
A(K) \geqq 2 A(C)-4 / 3 A(H)=A(T) .
$$

This completes the proof.

To prove that a relative Reuleaux triangle is the only body equivalent in width to $C$ with minimum area requires a little more argument. A sketch of the proof is as follows. If $K$ is such a body of minimum area, then equality must hold throughout (20). This means that $A(K,-K)=A\left(H^{\prime}\right)$ for a $C$-hexagon $H^{\prime}$ circumscribed about $K$. It follows that $A(-K, K)=A\left(H^{\prime}, K\right)$. If we let $p_{1}(\theta), p_{2}(\theta)$ be the support functions of $K$ and $H^{\prime}$ respectively, with origin at the center of $H^{\prime}$, and let $s_{1}$ denote arclength along $K$, the last equation implies that

$$
\int p_{1}(\theta+\pi) d s_{1}=\int p_{1}(\theta) d s_{1} .
$$

Equation (22) implies that $K$ must pass through 3 alternate vertices of $H^{\prime}$, from which readily follows the fact that $K$ is a relative Reuleaux triangle.

4. For any $n$-dimensional convex body $K$ we define a "coefficient of outer symmetry," $\mu(K)$, as follows. Let $S$ be a centrally symmetric convex body of minimum volume containing $K$. Then

$$
\mu(K)=\frac{V(K)}{V(S)},
$$

Thus $\mu(K) \leqq 1$, and $\mu(K)=1$ if and only if $K$ is centrally symmetric. Sharp lower bounds for $\mu(K)$ are not known for $n \geqq 3$; however, it is known that $\mu(K) \geqq 1 / 2$ if $K$ is 2 -dimensional, with equality holding if and only if $K$ is a triangle. A standing conjecture is that in $E_{n}, n \geqq 3, \mu(K) \geqq \mu(T)$, where $T$ is a simplex.

THEOREM 5. Let $B$ be a plane convex body of constant width 1 . Then $\mu(B) \geqq \mu(R)$, where $R$ is a Reuleaux triangle, and equality holds only if $B$ is a Reuleaux triangle.

Proof. Let $H$ be a regular hexagon circumscribed about $B$. 
Then, using Theorem 3, we have

$$
\mu(B) \geqq \frac{A(B)}{A(H)} \geqq \frac{A(R)}{A(H)}=\frac{\pi-\sqrt{3}}{\sqrt{3}}=.81 \cdots .
$$

where $R$ is a Reuleaux triangle of width 1 . On the other hand, any centrally symmetric convex set $S$ containing $R$ must also contain an equilateral triangle $T$ of side 1 and thus has area $\geqq 2 A(T)=A(H)$. Hence

$$
\frac{A(R)}{A(H)}=\mu(R)
$$

Equality can hold in (24) only if $A(B)=A(R)$, which happens only if $B$ is a Reuleaux triangle (see end of $\S 3$ ). This completes the proof.

It is known that any set $K$ of constant width in $E_{3}$ admits a regular circumscribed octahedron $J$ (see [7]). Suppose $K$ has constant width 1 , and let $S$ be a centrally symmetric set of minimum volume containing $K$. Then, using (6),

$$
\mu(K)=\frac{V(K)}{V(S)} \geqq \frac{\beta}{V(J)}=\frac{2 \beta}{\sqrt{3}} \approx .42 .
$$

Clearly one can obtain crude lower bounds, in this same fashion, in terms of $\lambda_{n}$ and the volume of some centrally symmetric "covering body" $J_{n}$ (one could, for example, use for $J_{n}$ a sphere of radius $\sqrt{n /(2 n+2))}$.

\section{REFERENCES}

1. A. S. Besicovitch, Minimum area of a set of constant width, Proceedings of Symposia in Pure Mathematics, Vol. 7, Convexity (Amer. Math. Soc., 1963), 13-14.

2. W. Blaschke, Einige Bemerkungen über Kurven und Flächen von konstanter Breite, Ber. Verh. sächs. Akad. Leipzig 67 (1915), 290-297.

3. - Konvexe Bereiche gegebener konstanter Breite und kleinsten Inhalts, Math. Annalen 76 (1915), 504-513.

4. H. G. Eggleston, A proof of Blaschke's theorem on the Reuleaux Triangle, Quart. J. Math. 3 (1952), 296-7.

5. - Convexity, Cambridge Univ. Press, Cambridge, 1958.

6. W. J. Firey, Lower bounds for volumes of convex bodies, Archiv der Math. 16 (1965), 69-74.

7. D. Gale, On inscribing $n$-dimensional sets in a regular $n$-simplex, Proc. Amer. Math. Soc. 4 (1953), 222-225.

8. T. Kubota and D. Hemmi, Some problems of minima concerning the oval, Jour. Math. Soc. Japan 5 (1953), 372-389.

9. H. Lebesgue, Sur le problème des isopérimètres et sur les domaines de largeur constante, Bull. Soc. Math. France, C. R. (1914), 72-76.

10. D. Ohmann, Extremal probleme für konvexe Bereiche der euklidischen Ebene, Math. Z. 55 (1952), 346-352. 
11. M. Sholander, On certain minimum problems in the theory of convex curves, Trans. Amer. Math. Soc. 73 (1952), 139-173.

12. M. Yaglom and V. G. Boltyanskii, Convex figures, GITTL, Moscow, 1951 (Russian); English transl., Holt, Rinehart and Winston, New York, 1961.

Received September 29, 1965.

University of California, Davis 



\section{PACIFIC JOURNAL OF MATHEMATICS}

\section{EDITORS}

\author{
H. SAMELSON \\ Stanford University \\ Stanford, California \\ J. P. JANS \\ University of Washington \\ Seattle, Washington 98105
}

\author{
J. DUGUNDJI \\ University of Southern California \\ Los Angeles, California 90007 \\ RICHARD ARENS \\ University of California \\ Los Angeles, California 90024
}

\section{ASSOCIATE EDITORS}
E. F. BECKENBACH
B. H. NeumanN
F. WOLF
K. YOSIDA

\section{SUPPORTING INSTITUTIONS}

\author{
UNIVERSITY OF BRITISH COLUMBIA \\ CALIFORNIA INSTITUTE OF TECHNOLOGY \\ UNIVERSITY OF CALIFORNIA \\ MONTANA STATE UNIVERSITY \\ UNIVERSITY OF NEVADA \\ NEW MEXICO STATE UNIVERSITY \\ OREGON STATE UNIVERSITY \\ UNIVERSITY OF OREGON \\ OSAKA UNIVERSITY \\ UNIVERSITY OF SOUTHERN CALIFORNIA
}

STANFORD UNIVERSITY

UNIVERSITY OF TOKYO

UNIVERSITY OF UTAH

WASHINGTON STATE UNIVERSITY

UNIVERSITY OF WASHINGTON

AMERICAN MATHEMATICAL SOCIETY CHEVRON RESEARCH CORPORATION TRW SYSTEMS

NAVAL ORDNANCE TEST STATION 


\section{Pacific Journal of Mathematics}

\section{Vol. 19, No. 1 \\ May, 1966}

A. R. Brodsky, The existence of wave operators for nonlinear equations... 1

Gulbank D. Chakerian, Sets of constant width................... 13

Robert Ray Colby, On indecomposable modules over rings with minimum condition....................................... 23

James Robert Dorroh, Contraction semi-groups in a function space ....... 35

Victor A. Dulock and Harold V. McIntosh, On the degeneracy of the Kepler

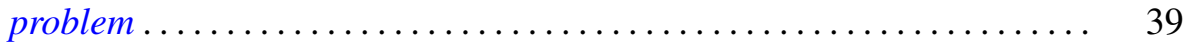

James Arthur Dyer, The inversion of a class of linear operators ......... 57

N. S. Gopalakrishnan and Ramaiyengar Sridharan, Homological dimension of Ore-extensions ................................. 67

Daniel E. Gorenstein, On a theorem of Philip Hall ................. 77

Stanley P. Gudder, Uniqueness and existence properties of bounded observables..................................... 81

Ronald Joseph Miech, An asymptotic property of the Euler function ....... 95

Peter Alexander Rejto, On the essential spectrum of the hydrogen energy and related operators ............................... 109

Duane Sather, Maximum and monotonicity properties of initial boundary

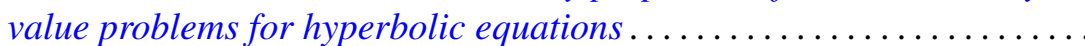

Peggy Strait, Sample function regularity for Gaussian processes with the parameter in a Hilbert space ........................... 159

Donald Reginald Traylor, Metrizability in normal Moore spaces ........... 175

Uppuluri V. Ramamohana Rao, On a stronger version of Wallis' formula ...............................

Adil Mohamed Yaqub, Some classes of ring-logics....... 